\title{
Supporting Health in the Hyogo Framework for Action-2
}

James J. James, MD, DrPH, MHA

$\mathrm{M}$ y planned editorial for this issue was to continue recounting the progress the Society of Disaster Medicine and Public Health has made toward achieving our original 2010 goals. ${ }^{1}$ Although this discussion is critically important in helping to define a discipline of disaster medicine and public health that is both professional and crossdisciplinary, that follow-up editorial is being deferred to the August issue for 2 reasons. The first is that 2 important meetings will be held in the near future that will better inform the discussion. The second reason is that the society has been asked to take an active role in supporting a growing initiative to better define health as a necessary element of the Hyogo Framework for Action-2 (HFA-2), as eloquently described by Burkle et al in an accompanying editorial. ${ }^{2}$

The HFA is an ongoing 10-year plan to lessen the impact of natural hazards that was endorsed by a United Nations (UN) General Assembly resolution (Resolution A/ RES/60/195) after the 2005 World Disaster Reduction Conference. At that time, the HFA outlined 5 priorities:

1. Ensure that disaster risk reduction is a national and a local priority with a strong institutional basis for implementation;

2. Identify, assess, and monitor disaster risks and enhance early warning;

3. Use knowledge, innovation, and education to build a culture of safety and resilience at all levels;

4. Reduce the underlying risk factors; and

5. Strengthen disaster preparedness for effective response at all levels.
Although efforts by, and input from, the health sector can be clearly inferred across all priority areas, this sector has not been clearly defined, health-related efforts have been limited, and no clear, overall plan has been developed. The exception to this deficit has been the highly regarded work performed under the Safe Hospitals Initiative of 2004, which was started in the Americas as essential to reducing underlying risk factors in disasters.

In developing a post-2015 HFA-2, health has been addressed as 1 of 12 areas that should be better incorporated. The UN General Assembly passed a resolution to hold the Third World Conference on Disaster Risk Reduction in Sendai City, Japan, from March 14-18, 2015, from which HFA-2 should emanate.

In support of this initiative, a meeting was held at George Washington University in Washington, DC, on May 21-22, 2014. The deliberations of that meeting also have been summarized by Burkle et al in this issue. From my perspective, this effort is critical in support of advancing the cause of global health security by enhancing the physical and mental resilience of people and communities worldwide. It is our sincere hope that other actions will follow, and that ever-increasing support for these goals can be garnered from the global health sector.

\section{REFERENCES}

1. James JJ. Disaster medicine and public health preparedness: a discipline for all health professionals—redux. Disaster Med Public Health Prep. 2014; 8(2):114-116.

2. Burkle FM, Egawa S, MacIntyre AG, Otomo Y, Beadling CW, Walsh JT. The 2015 Hyogo framework for action: cautious optimism. Disaster Med. Public Health Prep. 2014; 8(3):xxx-xxx. 